\title{
New Diamond Structures Observed by Aberration-corrected TEM
}

\author{
Jianguo $\mathrm{Wen}^{1}$, Duan $\mathrm{Luo}^{2}$, Liuxiang Yang ${ }^{3}$ and Hongxian Xie ${ }^{4}$
}

${ }^{1}$ Argonne National Laboratory, Lemont, Illinois, United States, ${ }^{2}$ Stanford University, Stanford, California, United States, ${ }^{3}$ Center for High Pressure Science and Technology Advanced Research, Lemont, Illinois, United States, ${ }^{4}$ Hebei University of Technology, Lemont, Illinois, United States

Color centers in diamond and silicon carbide have been dominantly used as solid-state quantum emitters due to their exceptional brightness, lattice-point dimension, and room temperature operation. Polytype $4 \mathrm{H}-\mathrm{SiC}$ and $6 \mathrm{H}-\mathrm{SiC}$, and the cubic $3 \mathrm{C}-\mathrm{SiC}$ are found to own desired properties to host Si-vacancy color centers. Other forms of diamond structures like hexagonal diamond (Lonsdaleite) or n-diamond could potentially offer better properties to host nitrogen-vacancy centers (NV-centers) due to their different band structures [1]. However, whether hexagonal diamond exists as a separate, pure material has been under debate [2]. Using aberration corrected TEM combined with XRD, we identified hexagonal diamond as a single phase. In addition, we found the consistence of two types of covalent bonds in hexagonal diamond.

There are two crystals forms of sp3-bonded carbon named diamond and Lonsdaleite. Each of the carbon atoms in both structures shares the same nearest and next nearest neighbor. The difference starts only from the second nearest neighbor, which cubic and hexagonal diamond has $\mathrm{ABC}$ and $\mathrm{ABAB}$ stackings, respectively. The hexagonal diamond sample is obtained using a single crystal graphite disk under wellcontrolled high pressure and temperature $(20 \mathrm{GPa}, 1400 \mathrm{~K})$ with a diamond anvil cell. Single crystal synchrotron x-ray diffraction on the recovered samples showed a hexagonal symmetry with space group P63/mmc. HRTEM images and selected-area electron diffraction also confirmed the obtained sample is a single phase of hexagonal diamond as shown in Fig. 1a and Fig. 1b. High-quality single-phase samples enable us to obtain carbon K-edge EELS spectra of the hexagonal diamond. Fig. 2c shows experimental carbon K-edge for hexagonal and cubic diamonds. Although both hexagonal and cubic diamonds have prominent peak structures (marked by I-IV) at $290.5-292 \mathrm{eV}, 298.5-299 \mathrm{eV}, 306-307 \mathrm{eV}$, and 329.5 $331 \mathrm{eV}$, the intensity distribution of the spectrum is apparently different between them. The major difference is the onset peak I higher for hexagonal diamond and the peak III shifting to the lower energy side. Since the electron states around the bottom of the conduction bands are affected by the long-range periodicity of a material, the clear difference in the spectra below $300 \mathrm{eV}$ is attributed to the different crystal structures between hexagonal and cubic diamonds. On the other hand, electron states at higher energy in the conduction band are mainly influenced by the distances to the first and second nearest neighboring atoms. The atomic distance of the first and second nearest neighbor atoms is almost the same for them, thus, it is expected to a similar spectrum above $300 \mathrm{eV}$. But, a clear peak shift for peak III is observed. This peak shift suggests a different bond structure for the hexagonal diamond. Aberrationcorrected HRTEM image shows that the hexagonal diamond has two different bonds, different from the single bond in cubic diamond. The observation of two bonds in the hexagonal diamond can explain the peak III shift. When lower synthesis temperature slightly, polytype diamonds are observed as shown in Fig. 2. These polytype diamonds consist of different intergrowth of cubic and hexagonal diamond [3]. 

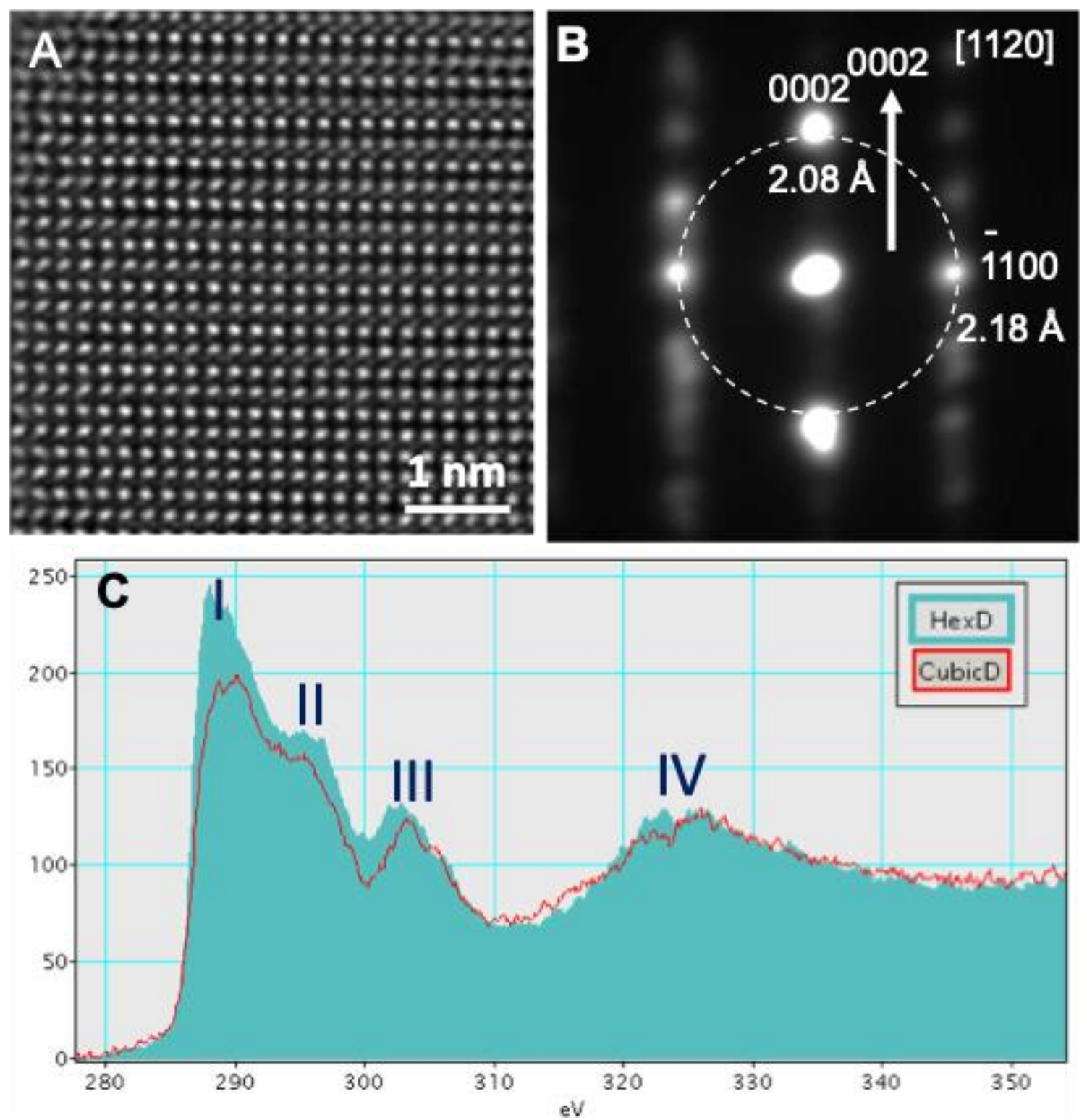

Figure 1. TEM observation of hexagonal diamond as a single phase. (a) HRTEM image (b) corresponding SAED pattern along [11-20] zone axis of hexagonal diamond. (b) A comparison of C-Kedge EELS between hexagonal and cubic diamond. 

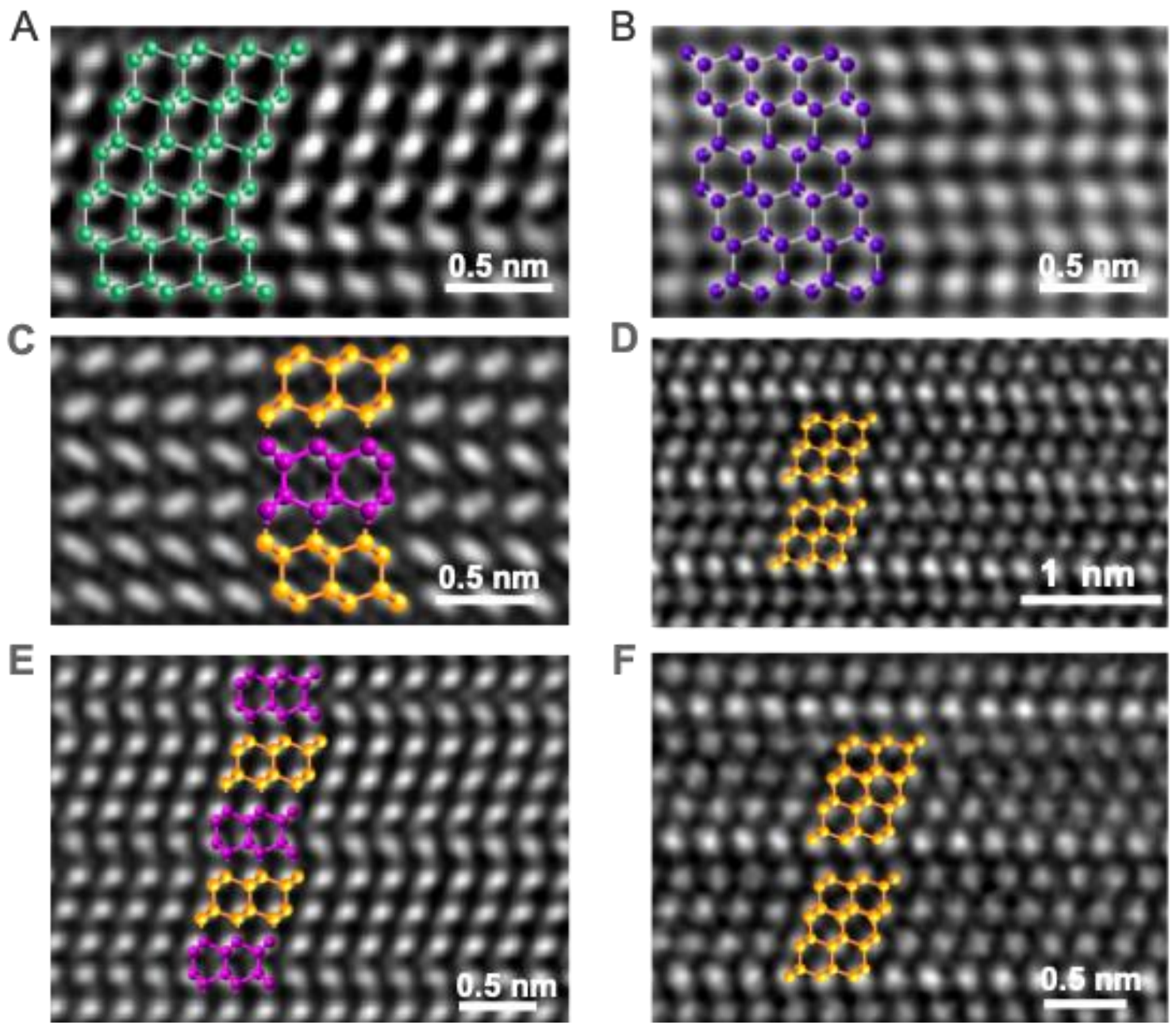

Figure 2. Intergrowth of cubic and hexagonal diamonds to form various types of polymorphs.

\section{References}

[1] Gali, Ádám, Nanophotonics 8.11 (2019): 1907-1943.

[2] Németh, Péter, et al. Nature Communications 5.1 (2014): 1-5.

[3] This work was performed at the Center for Nanoscale Materials, a U.S. Department of Energy Office of Science User Facility, and supported by the U.S. Department of Energy, Office of Science, under Contract No. DE-AC02-06CH11357. L.Y. also likes to thank the financial support from the National Nature Science Foundation of China (U51300340). 